\title{
Leishmania mexicana infection of the eyelid in a traveler to Belize
}

Joseph M. Vinetz ${ }^{1}$ and Lynn Soong ${ }^{2}$

${ }^{1}$ Division of Infectious Diseases, Department of Medicine, University of California,
San Diego School of Medicine, La Jolla, California; ${ }^{2}$ Departments of Microbiology
\& Immunology Pathology, University of Texas Medical Branch, Galveston, Texas, USA

A 50 year-old man, a United States resident, presented in Texas with a violaceous non-ulcerating lesion, involving the entire lower eyelid. The patient had traveled to a jungle area of Belize several hours drive from the capital city. Leishmania mexicana was isolated. The lesion only partially resolved after an initial course of sodium stibogluconate, requiring retreatment. At two years of follow-up, there was no relapse. The parasite isolated from the patient caused a progressive, non-ulcerating lesion in an experimental mouse footpad infection. This is an unusual case of cutaneous leishmaniasis in a traveler. Travelers must be educated about personal protective measures to prevent exotic infections acquired during travel.

Key Words: Leishmania mexicana, traveler, Belize.

Leishmaniasis is a vector-borne zoonotic disease caused by protozoan parasites transmitted by the bite of phlebotomine sand flies. In the Americas, four distinct syndromes of leishmaniasis affect humans: cutaneous, mucocutaneous, diffuse cutaneous, diffuse cutaneous and visceral. In the American continent, visceral leishmaniasis is caused by Leishmania chagasi/infantum; this is an important emerging opportunistic disease in patients with AIDS [1]. Mucocutaneous leishmaniasis is also becoming common [2]. Involvement of the mucosa of the nose, mouth and pharynx as highly disfiguring complications of L. brazilienses usually occurs late after the cutaneous form appears. Of relevance to this case report, travelers returning from Belize [3-5], including British soldiers [6,7], have had a relatively high risk of $L$. braziliensis infection, which mandates parenteral treatment to forestall late mucocutaneous complications. In the United States, leishmaniasis has taken on increased clinical importance because natural infections have been found in dogs (foxhounds) in a number of states [8]. With the recent incursions of the U.S. armed forces into the Middle East, many military personnel have returned with Old World cutaneous leishmaniasis, with more than 500 confirmed parasitological clinical cases. Among American troops serving in Iraq, 653 cases of cutaneous and two cases of visceral leishmaniasis were reported by the end of March 2004. According to U.S. sources, the number of infected American soldiers may have been higher, from 750 to 1,250, or even more, which would include nearly $1 \%$ of U.S. troops serving in Iraq in 2003-2004 [9-12]. Therefore, it is of clinical significance when cases of cutaneous leishmaniasis present within the United States,

Received on 10 May 2006; revised 29 July 2006.

Address for correspondence: Dr. Joseph Vinetz, M. D. University of California, San Diego School of Medicine, 9500 Gilman drive, 0640. La Jolla CA 92093-0649, USA. Phone: 1+858-822-4469. Fax: 1+858+534-6020. E-mail: jvinetz@ucsd.edu.

The Brazilian Journal of Infectious Diseases 2006;10(4):303-306. (C) 2006 by The Brazilian Journal of Infectious Diseases and Contexto Publishing. All rights reserved. especially in non-military personnel. Such infections most likely represent imported cases in travelers, but, given the autochthonous cases of canine leishmaniasis in the U.S., consideration must still be given to where patients acquired their infections.

\section{Case report}

A 50-year-old man traveled to a rural jungle area of Belize on vacation. He and his wife stayed seven days in a thatched-roof house and slept in hammocks without mosquito netting. Neither used insect repellant. One month after returning to his home on the Gulf coast of Texas, a non-ulcerated eye lesion developed (Figure 1). A biopsy was taken near the medial aspect of the lesion. A Giemsa-stained touch preparation of the biopsy showed intra-and extra-cellular amastigotes typical of Leishmania species (Figure 2). The biopsy specimen was cultured; parasites were visible seven days after the lesion was cultured. These parasites were identified as L. mexicana mexicana by isoenzyme testing at the United States Centers for Disease Control and Prevention, Atlanta, Georgia. Because of the reported prevalence of $L$. (Vianna) braziliensis in rural Belize, and because the lesion was progressive and in a cosmetically and functionally important anatomical area, the patient was given pentavalent antimony (sodium stibogluconate, Pentostam ${ }^{\mathrm{tm}}$ ) at a dose of $20 \mathrm{mg} / \mathrm{Kg}$ for 20 days. Only after treatment was completed was the infecting organism identified as L. m. mexicana. By the end of treatment, the lesion had more than $80 \%$ resolved. Two weeks after treatment was stopped, the lesion relapsed and continued to progress; Leishmania promastigotes again grew from an aspirate of the lesion. Antimony was restarted, continued for seven weeks, and the lesion resolved. Side effects from the first and second courses of antimonial treatment were primarily malaise and myalgia. Laboratory tests showed transient mild elevations in transaminases and serum lipase. An electrocardiogram showed mild flattening of the $\mathrm{T}$ waves. The patient was maintained on three additional months of oral itraconazole (200 mg /day). The patient's lesion resolved completely and healed without scarring. 


\section{Discussion}

We describe a case of New World cutaneous leishmaniasis involving the lower eyelid in a traveler to Belize. This case is particularly notable because of the unusual location of the infection, the relative refractoriness to treatment, and the species of the Leishmania parasite, L. m. mexicana, which caused the lesion.

New World cutaneous leishmaniasis typically presents as enlarging papules that progress to ulcerative lesions; the lack of ulceration in this case was atypical and led us to briefly consider that this patient had become infected by Trypanososoma cruzi, as manifested by Romana's sign, since T. cruzi is known to be endemic in Belize [13]. However, the appearance of the biopsy on histopathologic examination was not typical of Chagas disease, and the isolation of Leishmania $m$. mexicana from the lesion confirmed the diagnosis. Leishmaniasis affecting the eyelid has previously been reported in both Old World and New World cutaneous leishmaniasis, having been associated with L. (Vianna) braziliensis and L. major, but without culture confirmation [14-17].

In Belize, the most feared form of Leishmaniasis is caused by L. (Vianna) braziliensis, which is associated with mucocutaneous complications and relative refractoriness to therapy. Both cutaneous and mucocutaneous leishmaniasis due to L. (Vianna) braziliensis and L. m. mexicana have been observed as emerging health problems among travelers to Belize and other places in Central America [4].

Pentavalent antimonial drugs remain the treatment of choice for New World cutaneous and mucocutaneous leishmaniasis [3-5,7], when the lesions are clinically judged to require treatment. All cases of cutaneous leishmaniasis caused by L. (Vianna) braziliensis must be treated. Many cases of uncomplicated cutaneous lesions caused by L. $\mathrm{m}$. mexicana will self-resolve. Lesions on the face, the presence of large or many lesions, or progressive, non-healing lesions also necessitate treatment. Liposomal amphotericin B for $L$. (Vianna) braziliensis has recently been reported to be successful [18], but it is also associated with relapse [5]. Itraconazole was not found effective in primary treatment of New world mucocutaneous leishmaniasis in a small controlled clinical trial [19], although anecdotal evidence suggests that this drug may have some utility in the treatment of New World cutaneous leishmaniasis [20,21], particularly in consolidation or maintenance therapy, as seen in our case. Local, topical treatment with photodynamic therapy [22], paromomcym [23], and the immune stimulator imiquimod [2426] have also been shown to be effective treatments for $L$. (Vianna) braziliensis-related New World cutaneous leishmaniasis.

Our patient had a poor response to initial therapy. Two factors could have contributed to this slow clinical cure. The infecting strain of the parasite, L. mexicana mexicana, may have had intrinsic refractoriness to treatment with sodium stibogluconate. Since it is well known that the patient must develop effective T cell immunity in order to cure a lesion, whether spontaneously or in the presence of drug treatment, our patient may have had some genetic basis for his relative inability to form active delayed-type hypersensitivity. Precise identification of infecting leishmanial parasites and their in vitro drug susceptibility, if available in a timely way, would be key for guiding therapy and indicating the risk of mucocutaneous disease.

Given the recently-observed autochthonous transmission of Leishmania infections among dogs in the United States [8], we considered whether our patient acquired his infection in Texas rather than during his travel to Belize. The patient lives in suburban Houston, where there has not been any observed cutaneous leishmaniasis. Given the travel history, we believe that it is most likely that the patient acquired his infection in the Belize jungle.

The patient's clinical presentation was characterized by a lack of ulceration and the finding of large numbers of intraand extra-cellular organisms. Cutaneous leishmaniasis is typified by its progression to ulceration over time, so the lack of ulceration over several months of progressive infection is striking. This clinical finding is consistent with the lack of development of cell mediated immunity, which is necessary for spontaneous self-cure in this syndrome.

Experimental inoculation of mouse footpads showed that the $L$. m. mexicana isolate from the patient had a propensity not to induce ulcerative disease, even up to 10 weeks after infection, in a well-established mouse model of cutaneous leishmaniasis (Figure 3). The mouse model using this isolate may be useful for determining mechanisms by which Leishmania parasites induce ulceration in the protective $\mathrm{T}$ cell responses. This patient's isolated Leishmania was cultured as axenic amastigotes in vitro, making detailed proteomic and immunological investigations of pathogenesis and protective immunity feasible. Understanding such mechanisms may have clinical utility in modifying sequels of scarring and may also yield insight into how to appropriately use immunomodulators in the management of cutaneous and mucocutaneous leishmaniasis.

\section{Acknowledgments}

We thank Barbara Herwaldt, M.D. of the U.S. Centers for Disease Control, Atlanta, Georgia, USA, the staff of the CDC investigational drug service and the CDC Leishmania diagnostic laboratory for their help in diagnosing and managing this case. We also thank the nursing staff of the University of Texas Medical Branch, Galveston, Texas, USA, and the clinical house staff and fellows, particularly Bruno Granwehr, M.D. and Miguel Montes-Walters of the Division of Infectious Diseases at UTMB, who helped in the care of the patient. 
Figure 1. Patient with left lower eyelid progressive swelling, erythema, without ulceration. The puncture at the medial edge of the lesion is the biopsy site.

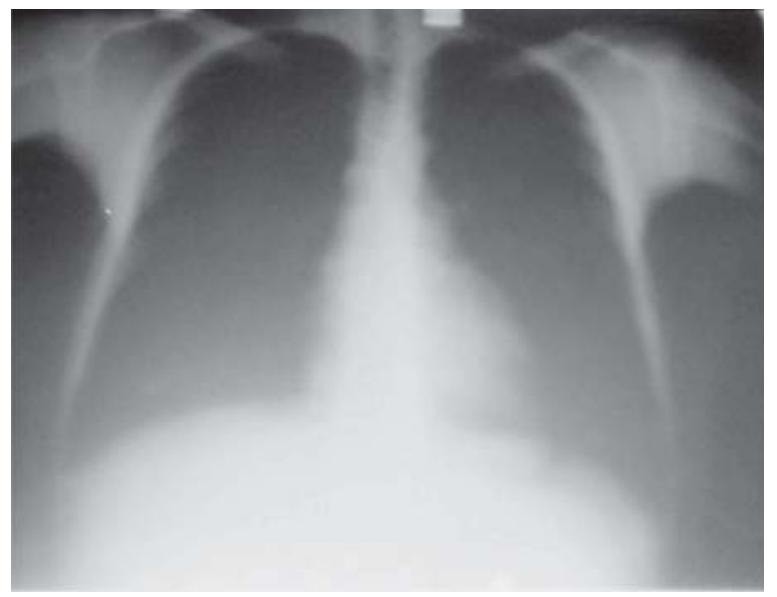

Figure 2. Giemsa-stained examination of a touch preparation of the patient's lesion (400X). At left, mononuclear cells contain large clusters of leishmania amastigotes with scattered extracellular amastigotes visible. At right, many extracellular amastigotes are apparent, showing the poor T cell response to this infection. Kinetoplasts, extranuclear collections of minicircle DNA, are well-visualized in many parasites.

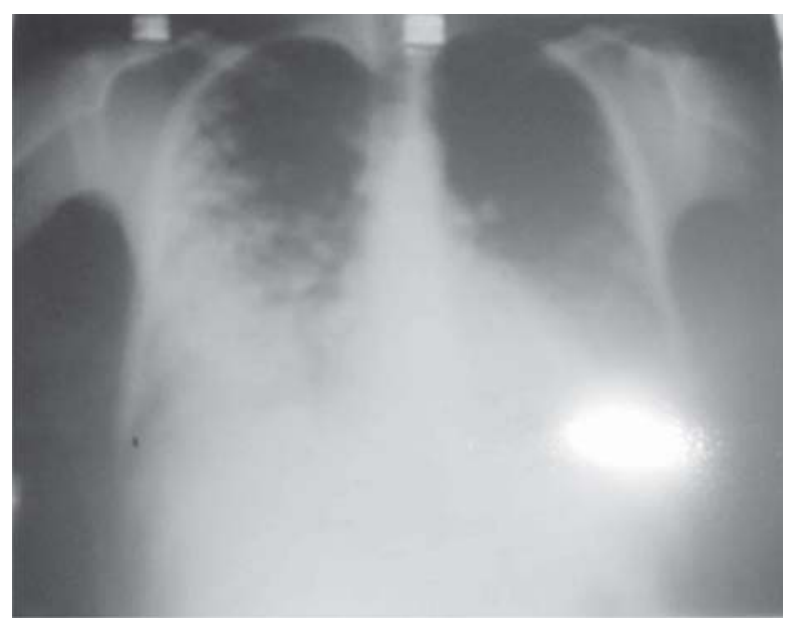

Figure 3. Appearance of the hind foot of a C57BL/6 mouse at 10 weeks after subcutaneous/intradermal inoculation of the patient's Leishmania isolate. The lesion was progressive, non-healing, and had a remarkable absence of ulceration.

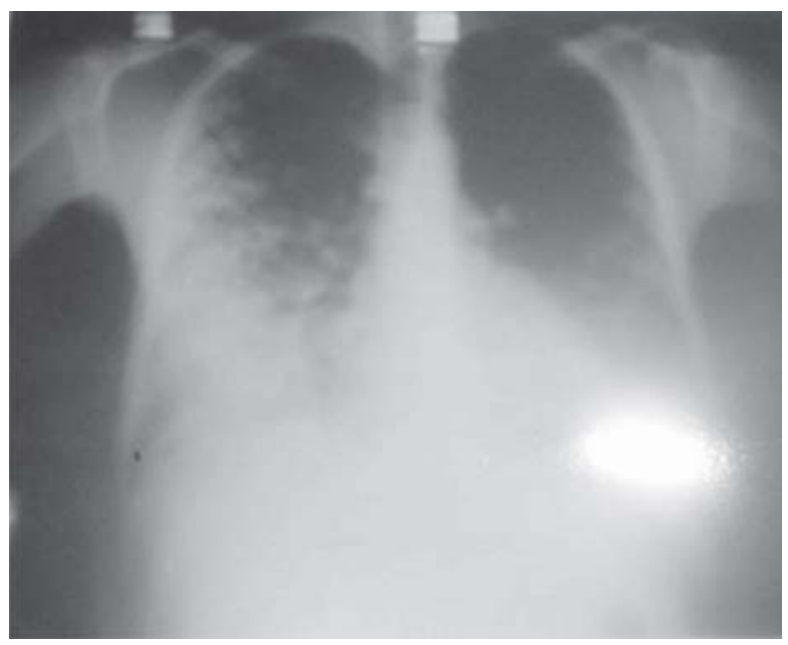




\section{References}

1. Desjeux P. Leishmaniasis. Nat Rev Microbiol 2004;2:692.

2. Herwaldt B. Leismaniasis. Lancet 1999;354:1191-11.

3. Marsden P.D. Mucosal Leishmaniasis ("espundia” Escomel, 1911), Trans R Soc Trop Med Hyg 1986;80:859-76.

4. Lawn S.D., Whetham J., Chiodini P.L., et al. New World mucosal and cutaneous leishmaniasis: an emerging health problem among British travellers. QJM 2004;97:781-8.

5. Zeegelaar J.E., Steketee W.H., van Thiel P.P.A.M., et al. Changing pattern of imported cutaneous leishmaniasis in the Netherlands. Clin Exp Dermatol 2005;30:1-5.

6. Hepburn N.C., Tidman M.J., Hunter J.A. Cutaneous leishmaniasis in British troops from Belize. Br. J Dermatol 1993;128;63-8.

7. Palmer R.A., Tran D., Hepburn N.C., Ashton R.E. The management of cutaneous leishmaniasis from Belize. Clin Exp Dermatol 2001;26:16-20.

8. Enserink M. Infectious diseases. Has leishmaniasis become endemic in the U.S.? Science 2000;290:1881-3.

9. Update: Cutaneous leishmaniasis in U.S. military personnelSouthwest/Central Asia, 2002-2004. MMWR Morb Mortal Wkly Rep 2004;53:264-5.

10. Cutaneous Leishmaniasis in U.S. Military personnel Southwest/Central Asia, 2002-2003. MMWR Morb Mortal Wkly Rep 2003;52:1009-12.

11. Magil A.J., Grol M., Gasser R.A., Jr., Sun W., Oster C.N. Visceral infection caused by Leishmania tropica in veterans of Operation Desert Storm. N Engl J Med 1993;328:1383-7.

12. Martin S., Gambel J., Jackson J., et al. Leishmaniasis in the United States military. Mil. Med 1998;163:801-7.

13. Jaramillo R., Bryan J.P., Schur J., Pan A.A. Prevalence of antibody to Trypanosoma cruzi in three populations in Belize. Am J Trop Med Hyg 1997;57:298-301.

14. Chu F.C., Rodrigues M.M., Cogan D.G., Neva F.A. Leishmaniasis affecting the eyelids. Arch Ophthalmol 1983;101:84-91.
15. Rentsch F.J. Cutaneous leishmaniasis (Oriental sore) of the lids. Klin Monatsbl Augenheilkd 1980;177:75-9.

16. Ferry A.P. Cutaneous leishamaniasis (oriental sore) of the eyelid. Am J Ophthalmol 1977;84:349-54.

17. Morgan G. Case of cutaneous leishmaniasis of the lid. Br J Ophthalmol 1965;49:542-6.

18. Brown M., noursadeghi M., Boyle J., Davidson R.N. Successful liposomal amphotericin B treatment of Leishmania braziliensis cutaneous leishmaniasis. Br J Dermatol 2005;153:203-5.

19. Calvopina M., Guevara A.G., Armijos R.X., et al. Itraconazole in the treatment of New World mucocutaneous leishmaniasis. Int J Dermatol 2004;43:659-63.

20. Borelli D. A clinical trial of itraconazole in the treatment of deep mycoses and leishmaniasis. Rev Infect Dis 1987; Suppl 1 S57-S63.

21. Dogra J., Saxena V.N. Itraconazole and leishmaniasis: a randomized double-blind trial in cutaneous disease. Int $\mathrm{J}$ Parasitol 1996;26:1413-5.

22. Gardlo K., Horska Z., Enk C.D., et al. Treatment of cutaneous leishamaniasis by photodynamic therapy. J Am Acad Dermatol 2003;48: 893-6.

23. Gonçalves G.S., Fernandes A.P., Souza R.C., et al. Activity of a paromomycin hydrophilic formulation for topical treatment of infectious Leishmania (Leishmania) amazonnensis and Leishmania (Viannia) braziliensis. Acta Trop 2005;93:161-7.

24. Miranda-Verastegui C., Llanos-Cuentas A., Arevalo I., et al. Randomized, double-blind clinical trial of topical imiquimod $5 \%$ with parenteral meglumine antimoniate in the treatment of cutaneous leishmaniasis in Peru. Clin Infect Dis 2005;40:1395-403.

25. Seeberger J., Daoud S., Pammer J. Transient effect of topical treatment of cutaneous leishmaniasis with imiquimod. Int $\mathrm{J}$ Dermatol 2003;42:576-9.

26. Arevalo I., Ward B., Miller R., et al. Successful treatment of drug-resistant cutaneous leishmaniasis in humans by use of imiquimod and immunomodulator. Clin Infect Dis 2001;33:1847-51. 